\title{
Communication \\ Evaluation of Four Lateral Flow Assays for the Detection of Legionella Urinary Antigen
}

\author{
Alicia Y. W. Wong ${ }^{1, *}$, Alexander T. A. Johnsson ${ }^{2}$, Aina Iversen ${ }^{1,3}$, Simon Athlin ${ }^{4}$ (D) and Volkan Özenci ${ }^{1,2, *}$ \\ 1 Division of Clinical Microbiology, Department of Laboratory Medicine, Karolinska Institutet, \\ 14186 Stockholm, Sweden; aina.iversen@sll.se \\ 2 Department of Clinical Microbiology, Karolinska University Hospital, Huddinge, \\ 14186 Stockholm, Sweden; alexander.johnsson@sll.se \\ 3 Department of Clinical Microbiology, Karolinska University Hospital, Solna, 17176 Stockholm, Sweden \\ 4 School of Medical Sciences, Faculty of Medicine and Health, Örebro University, 70182 Örebro, Sweden; \\ simon.athlin@oru.se \\ * Correspondence: alicia.wong@ki.se (A.Y.W.W.); volkan.ozenci@ki.se (V.Ö.)
}

Citation: Wong, A.Y.W.; Johnsson,

A.T.A.; Iversen, A.; Athlin, S.; Özenci,

V. Evaluation of Four Lateral Flow Assays for the Detection of Legionella Urinary Antigen. Microorganisms 2021, 9, 493. https://doi.org/ 10.3390/microorganisms 9030493

Academic Editor: Janet E. Stout

Received: 2 February 2021

Accepted: 23 February 2021

Published: 26 February 2021

Publisher's Note: MDPI stays neutral with regard to jurisdictional claims in published maps and institutional affiliations.

Copyright: (c) 2021 by the authors. Licensee MDPI, Basel, Switzerland. This article is an open access article distributed under the terms and conditions of the Creative Commons Attribution (CC BY) license (https:// creativecommons.org/licenses/by/ $4.0 /)$.

\begin{abstract}
Urinary antigen tests (UATs) are often used to diagnose Legionnaires' disease as they are rapid and easy to perform on readily obtainable urine samples without the need for specialized skills compared to conventional methods. Recently developed automated readers for UATs may provide objective results interpretation, especially in cases of weak result bands. Using 53 defined patient urine samples, we evaluated the performance of the BinaxNOW Legionella Antigen Card (Abbott), ImmuView S. pneumoniae and Legionella (SSI Diagnostica), STANDARD F Legionella Ag FIA (SD Biosensor), and Sofia Legionella FIA (Quidel) simultaneously with their respective automated readers. Automatic and visual interpretation of result bands were also compared for the immunochromatography-based BinaxNOW and ImmuView UATs. Overall sensitivity and specificity of Legionella UATs were 53.9-61.5\% and 90.0-94.9\%, respectively. All four UATs successfully detected all samples from L. pneumophila serogroup 1-positive patients, but most failed to detect samples for Legionella spp., or other serogroups. Automatic results interpretation of results was found to be mostly concordant with visual results reading. In conclusion, the performance of the four UATs were similar to each other in the detection of Legionella urinary antigen with no major difference between automated or visual results reading.
\end{abstract}

Keywords: Legionella pneumophila; Legionella antigen; pneumonia; urinary antigen test (UAT); BinaxNOW; ImmuView; STANDARD F; Sofia

\section{Introduction}

Legionnaires' disease is an uncommon but severe pneumonia-type illness caused by Legionella spp. In 2018, there were almost 10,000 cases of Legionnaire's disease in the USA [1] and 10,672 confirmed cases in the EU/EEA [2]. It is estimated that Legionnaires' disease is fatal in around 1 in 10 cases [3,4]. Although culture of clinical lower respiratory tract specimens remains the gold standard method for diagnosis of Legionnaires' disease, the most commonly utilized diagnostic tool is the use of urinary antigen tests (UATs). Unlike culture-based methods that require 3-5 days and requires training in Legionella colony identification, and the use of specific procedures and specialized media for isolating Legionella from patient sputum, UATs are easy to perform and results are obtained within 15-20 min, hence speeding up patient diagnosis [5]. It is also common to detect Legionella infection by PCR [5]. However, lab staff performing PCR must be specially trained for molecular techniques, and obtaining sputum samples for culture and/or PCR is more challenging compared to the more readily available urine.

There are currently 58 species and more than 70 serogroups known of Legionella, of which 30 species have been reported to cause human infection [4]. The vast majority of 
UATs are focused on detecting Legionella pneumophila serogroup 1, which is the causative agent for $50-80 \%$ of Legionnaires' disease cases [5]. There are also a few UATs reported in literature that are not confined to detecting only L. pneumophila serogroup 1 [6-10].

Hitherto published studies on rapid Legionella UATs for detection of L. pneumophila serogroup 1 reported sensitivities and specificities ranging between $55.5-96.0 \%$ and $95.6-$ $100 \%$, respectively, using different technologies for antigen detection [8-13]. Immunochromatography (ICT)-based UATs produce a visible result band for interpretation, while fluorescent immunoassay (FIA)-based UATs require the use of an automated reader for interpretation. There is a possibility of obtaining visually faint result bands on ICT-based UATs that could be easily missed [7], which could lead to false-negative results.

Automated readers for ICT-based UATs that have been developed recently may provide more objective readings particularly in the case of weak result bands. To our knowledge, the potential benefits of using automated readers for ICT-based UATs has not been yet explored, hence the present study aims to compare the performance of four Legionella UATs with automated reading on urine samples from patients with and without PCR-verified Legionella infection. Here, we compare two ICT-based UATs, the BinaxNOW Legionella Antigen Card (BinaxNOW) (Abbott, Chicago, IL, USA) and ImmuView S. pneumoniae and L. pneumophila (ImmuView) (SSI Diagnostics, Hillerød, Denmark), and two FIA-based UATs, the STANDARD F Legionella FIA (STANDARD F) (SD Biosensor, Gyeonggi, Korea) and Sofia Legionella FIA (Sofia) (Quidel Corporation, San Diego, CA, USA) were evaluated simultaneously. In addition, the automatically read results obtained with ICT-based UATs were also compared to visually read results.

\section{Materials and Methods}

\subsection{Samples}

Urine samples from adult patients ( $\geq 18$ years old) were collected at the Department of Clinical Microbiology at Karolinska University Hospital, Sweden and the Department of Laboratory Medicine at Örebro University Hospital, Sweden. Urine samples from patients who were positive for Legionella by PCR were used as positive cases. Urine samples from patients that had infections of non-Legionella etiology were used as negative controls (Figure 1, Tables S1 and S2). All samples were anonymized after culture results had been retrieved. Samples were stored at $-20{ }^{\circ} \mathrm{C}$ or colder and were thawed in room temperature prior to testing. One sample per patient were used and each sample was tested with all four UATs (BinaxNOW, ImmuView, STANDARD F, and Sofia).

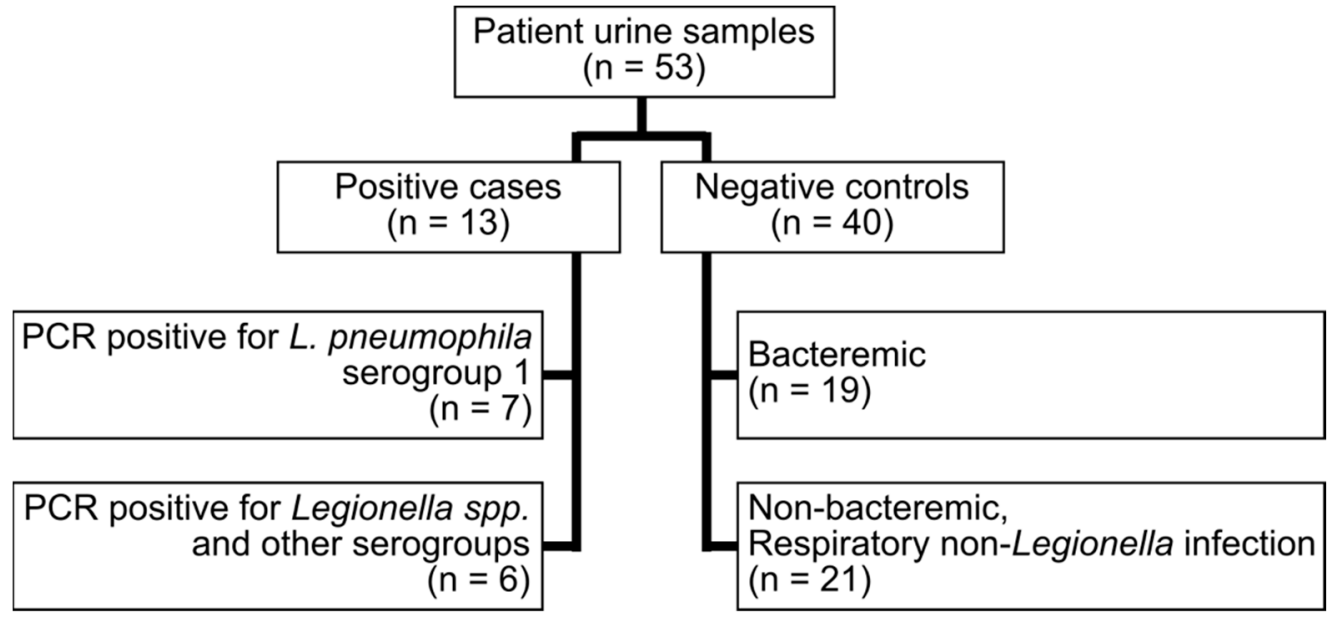

Figure 1. Patient urine samples included in the study. 


\subsection{Urinary Antigen Tests}

UATs from the four manufacturers were used together with their respective readers. The four UATs included in the present study were the BinaxNOW together with the DIGIVAL instrument, the ImmuView together with the ImmuView Reader, the STANDARD F together with the STANDARD F200 Analyzer, and the Sofia together with the Sofia Fluorescent Immunoassay Analyzer. For the BinaxNOW, STANDARD F, and Sofia, samples were incubated and read by the readers on "Walk Away" mode. UAT setup procedures are briefly described as follows and interpretations were performed according to each manufacturer's instructions. BinaxNOW setup included dipping the included swab into the sample, inserting it into the sample card, adding two drops of kit-included reagent buffer, and then inserting the sample card into the reader for incubation and interpretation as per manufacturer's instructions. For ImmuView, three drops of sample were added to the included polypropylene tube followed by addition of two drops of the kit-included reagent buffer, and gently mixed. The UAT strip was then inserted into the tube, and incubation was timed manually before the UAT was inserted into the reader for interpretation. Sample incubation time was $15 \mathrm{~min}$ for both the BinaxNOW and ImmuView. Test results for BinaxNOW and ImmuView were also visually interpreted by two independent researchers. The appearance of a correctly colored band that was visible to the naked eye was considered as a positive result. If the visual interpretations of the UAT differed between researchers, the result that was consistent with automated reading was used. For STANDARD F and Sofia, the protocol had no reagent buffer thus only the sample was introduced to the sample well on the cassette using the kit-included fixed volume dropper, and the cassettes were then inserted into their respective reader for incubation and interpretation as per manufacturers' instructions. Samples were run simultaneously with each of the four UATs, and the results provided by the readers were noted. Sample incubation time was $15 \mathrm{~min}$ for STANDARD F, and $10 \mathrm{~min}$ for the Sofia. The readers indicated if the sample result was positive or negative for Legionella and if the test was valid. If a result obtained for a sample was invalid or the reader displayed an error message, the sample was re-analyzed using a new UAT. The STANDARD F200 Analyzer also indicated the cut-off index (COI) value along with the sample result.

\subsection{Ethical Permission}

The samples that were used in the study were leftover samples that were submitted to the laboratory. The samples were anonymized and information on patient characteristics was not used. Therefore ethical permission was not needed for the study.

\subsection{Statistical Analysis}

Sensitivities and specificities of the UATs were determined, and the confidence intervals $(\mathrm{CI})$ were computed using the Wilson-Brown method. Statistical calculations for the Wilson-Brown method were performed using Prism 8 (GraphPad Software LLC, San Diego, CA, USA). A CI of $95 \%$ was used for statistical precision.

\section{Results}

\subsection{Sample Characteristics}

A total of 53 samples were analyzed, 13 were positive cases and 40 negative controls (Figure 1, Tables S1 and S2).

\subsection{Sensitivity of Legionella UATs}

All UATs successfully detected Legionella antigen in samples from four patients with confirmed L. pneumophila serogroup 1 infection, and three patients with non-serogrouped L. pneumophila (Table 1). The UATs did not detect Legionella antigen in samples from patients infected with other Legionella spp. (Table 2, Table S1). One sample from a patient with PCR-confirmed L. pneumophila serogroup 6 was detected by ImmuView only and yielded an invalid result with Sofia. 
Table 1. Sensitivity of four Legionella urine antigen assays on samples *.

\begin{tabular}{cccc}
\hline Assay & Positive Result & Negative Result & Sensitivity (95\% CI) \\
\hline BinaxNOW & 7 & 0 & $100.0(64.6-100.0)$ \\
ImmuView & 7 & 0 & $100.0(64.6-100.0)$ \\
STANDARD F & 7 & 0 & $100.0(64.6-100.0)$ \\
Sofia & 7 & 0 & $100.0(64.6-100.0)$ \\
\hline
\end{tabular}

* Urine samples from patients with PCR-confirmed L. pneumophila infection.

Table 2. Positivity of four Legionella urine antigen assays on samples from patients with PCRconfirmed Legionella infection by species other than Legionella pneumophila serogroup 1 *.

\begin{tabular}{ccccc}
\hline Assay & Positive Result & Negative Result & Invalid/Error & $\begin{array}{c}\text { Positivity Rate } \\
\text { (95\% CI) }\end{array}$ \\
\hline BinaxNOW & 0 & 6 & 0 & $0.0(0.0-39.0)$ \\
ImmuView & 1 & 5 & 0 & $16.7(0.9-56.4)$ \\
STANDARD F & 0 & 6 & 0 & $0.0(0.0-39.0)$ \\
Sofia & 0 & 5 & 1 & $0.0(0.0-39.0)$
\end{tabular}

* L. longbeachae $(n=2)$, L. bozemanii $(n=2)$, L. pneumophila serogroup $6(n=1)$, Legionella spp. $(n=1)$. \%endtabular

\subsection{Specificity of Legionella UATs}

The specificities of the four Legionella UATs ranged from 90.0-94.9\% (Table 3). A total of six negative controls yielded false positive results in one or more UATs (Table 4). For one sample (N15, Table 4, Table S2), the reader indicated insufficient sample volume error in all four attempts with the STANDARD F UAT. These cassettes from four failed attempts were inspected, and it was observed that the sample failed to reach the end of the test strip in the cassettes.

Table 3. Specificities of four Legionella urine antigen assays.

\begin{tabular}{ccccc}
\hline Assay & $\begin{array}{c}\text { Positive } \\
\text { Result }\end{array}$ & Negative Result & Invalid/Error & $\begin{array}{c}\text { Specificity } \\
\text { (95\% CI) }\end{array}$ \\
\hline BinaxNOW & 3 & 37 & 0 & $92.5(80.1-97.4)$ \\
ImmuView & 4 & 36 & 0 & $90.0(77.0-96.0)$ \\
STANDARD F & 2 & 37 & 1 & $94.9(83.1-99.1)$ \\
Sofia & 3 & 37 & 0 & $92.5(80.1-97.4)$ \\
\hline
\end{tabular}

Table 4. False positive and discordant test results in six patients without Legionella infection.

\begin{tabular}{|c|c|c|c|c|c|c|}
\hline Sample & $\begin{array}{l}\text { Blood Culture } \\
\text { Result }\end{array}$ & $\begin{array}{c}\text { Respiratory } \\
\text { Culture Result }\end{array}$ & BinaxNOW & ImmuView & STANDARD F & Sofia \\
\hline N6 & B. fragilis & $\begin{array}{c}\text { Negative } \\
\text { (normal flora) }\end{array}$ & Negative & Positive & Negative & Negative \\
\hline N7 & E. coli & $\begin{array}{c}\text { Negative } \\
\text { (normal flora) }\end{array}$ & Negative & Positive & Positive & Positive \\
\hline N8 & E. coli & $\begin{array}{c}\text { Negative } \\
\text { (normal flora) }\end{array}$ & Positive & Negative & Negative & Negative \\
\hline N14 & K. pneumoniae & $\begin{array}{c}\text { Negative } \\
\text { (normal flora) }\end{array}$ & Negative & Positive & Negative & Positive \\
\hline N15 & P. mirabilis & $\begin{array}{c}\text { Negative } \\
\text { (normal flora) }\end{array}$ & Positive & Negative & $\begin{array}{l}\text { Insufficient } \\
\text { volume error }\end{array}$ & Negative \\
\hline N20 & Negative & C. albicans & Positive & Positive & Positive & Positive \\
\hline
\end{tabular}




\subsection{Agreement between Visual and Automatic Reading of UATs}

The visual results interpretations of the BinaxNOW and ImmuView UATs by the two researchers were $100 \%$ in agreement with each other. However, there were some inconsistencies observed between the visual and automatic reading results in two of the samples with the BinaxNOW UAT and with the ImmuView UAT. For the BinaxNOW UAT, two samples (N8 and N15, Table 4, Table S2) yielding a positive result by automatic reading were interpreted as negative visually by the two researchers. For the ImmuView UAT, one Legionella positive sample ( $\mathrm{P} 4$, Table S1) also yielded a positive result for S. pneumoniae on the same test strip by automatic reading, but was negative by visual. As this urine sample was tested, both BinaxNOW and ImmuView yielded very strong test results for Legionella antigen, which may have interfered with the automated interpretation of pneumococcal antigen detection by the ImmuView reader (Figure 2). Another sample, used as a negative control (N14, Table 4, Table S2), yielded an invalid visual test result with the ImmuView as both the test line for Legionella and the control line turned purple, while the automatic reading yielded a positive result. When the sample was re-analyzed, the visual test result was negative while the automatic result was positive.

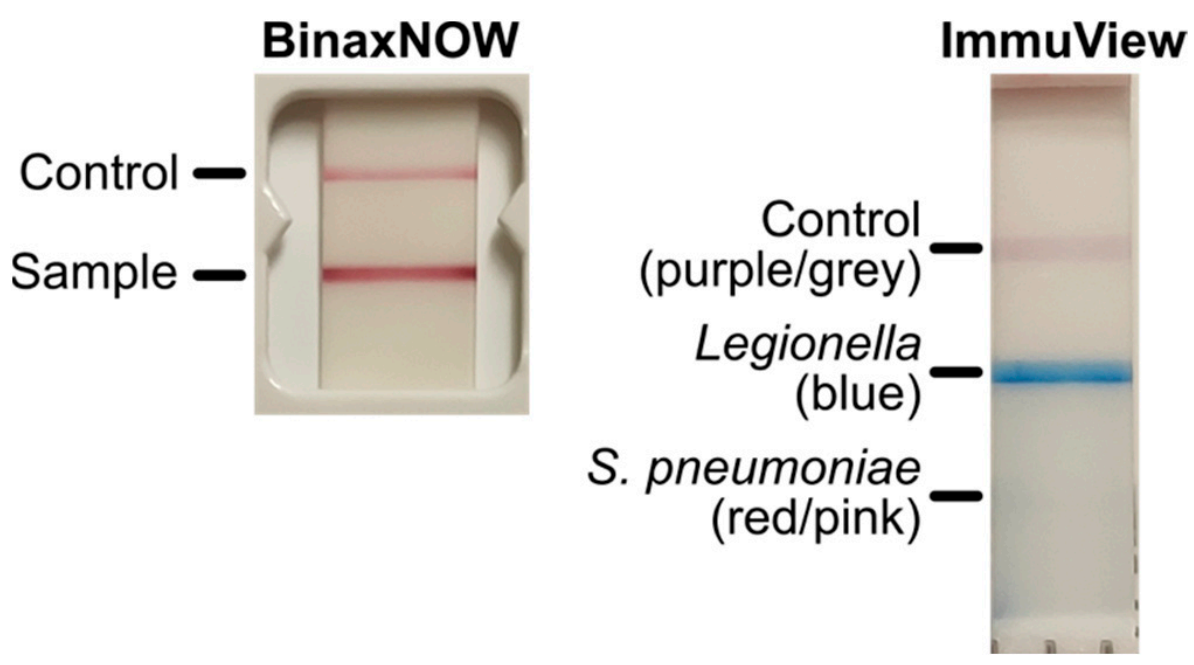

Figure 2. BinaxNOW and ImmuView test results for positive case P4.

\section{Discussion}

Using a rapid UAT for diagnosing Legionnaires' disease is recommended by pneumonia management guidelines due to the simplicity to perform the test on urine samples. The assay turn-around time is within minutes, with high accuracy in detecting L. pneumophila serogroup 1 antigen in previous studies [5]. Therefore, it is utterly important to compare the available commercial UAT methods for detection of Legionella spp. in urine. The present study compares for the first time four UATs using clinical samples with their respective automated interpretation, showed that the UATs performed similarly to each other. All UATs examined detected Legionella urinary antigen in samples from patients with L. pneumophila serogroup 1 infection. The specificity of the UATs were $90-95 \%$. In addition, results were found to be mostly concordant between automatic and visual interpretation of the BinaxNOW and ImmuView UATs.

All four UATs yielded negative results on samples that were non-L. pneumophila serogroup 1 Legionella spp. positive, which is in line with the test validation by the manufacturers, and has been demonstrated in previous studies [7,8]. As for the detection of other L. pneumophila serogroups, a sample from a patient infected with L. pneumophila serogroup 6 tested positive in the ImmuView despite the detection of other serogroups was not explicitly stated in the ImmuView product information. The BinaxNOW UAT product information has stated that the UAT is unable to detect urinary antigens of other L. pneumophila serogroups or Legionella spp., although past studies have shown that it is 
capable of detecting urinary antigens of other L. pneumophila serogroups [7,8]. In contrast, the product information for the STANDARD F and Sofia UATs mentioned the potential for both to detect the antigens of other L. pneumophila serogroups (serogroups 3, 5, 6, and 8 for the STANDARD F; serogroups 3, 4, and 6 for the Sofia). However, the L. pneumophila serogroup 6 sample tested negative on both UATs in the present study. Nevertheless, the detection non-L. pneumophila serogroup 1 infection is unreliable and underscores the importance of not relying solely on UATs for diagnosis of Legionnaires' disease as they would miss $20-50 \%$ of Legionnaires' disease cases that are caused by other Legionella species and serogroups [5].

The specificities of the UATs were found to be slightly lower compared to what was reported in other studies, perhaps due to the small sample size. Interestingly, we observed that $5 / 6$ of the samples that tested false positive in one or more of the UATs were from patients with Gram-negative bacteremia. To our knowledge, false positivity of UATs in cases of non-Legionella bacteremia has not been previously reported. The underlying reason for this finding is not known. The last false positive result was a sample from a patient without bacteremia but was respiratory culture positive for $C$. albicans tested positive in all four UATs. This patient's bronchoalveolar lavage sample was PCR-negative for Legionella. As false positivity may occur if the patient had a past Legionella infection since Legionella antigens can be excreted in urine for as long as a year [14], it is not possible to exclude the possibility of resolved any past Legionella infection that could give rise to antigen positivity in the urine. False positivity with Legionella UATs have also been previously demonstrated in patients with serum sickness [15,16], and for samples of this nature, boiling is a possible way to eliminate false positives due to the heat sensitive nature of rheumatoid-like factors compared to the heat resistance of bacterial antigens [16]. Contradictory evidence for boiling of samples exist in the literature. For instance, it was shown that boiling samples increased the specificity of the Sofia UAT by 2.3\% (compared to results obtained by BinaxNOW) [17]; however, another study showed that while boiling did not affect the sensitivity of the ImmuView UAT, the sensitivity of the BinaxNOW was worse in boiled samples compared to unboiled samples tested for Legionella antigen [7]. Sample boiling to confirm positive results is recommended by some manufacturers of UATs, but was not recommended by the BinaxNOW and STANDARD F UATs. In the present study, all samples were tested by all four tests in similar conditions, therefore, the urine samples were not boiled.

The interpretation of colorimetric bands on ICT-based assays could be subjective compared to FIA-based assays that are read automatically [17]. In the present study, 3 out of 106 total readings of ICT-based UAT results obtained on automated readers were in line with visually read results. In addition, one of the negative control samples (N6, Table 4, Table S2) that tested positive for Legionella with the ImmuView UAT by both automatic reading and visually was noted to have a visually faint Legionella band by both researchers. While this was not judged as an inconsistency of results between automated and visual readings, it indicated that automated readers could be more sensitive in detecting faint result bands that otherwise might be missed if only a visual reading was done.

The STANDARD F Legionella UAT was unique in this study as the only UAT that presents a COI value as a way to quantify the concentration of Legionella antigen in the sample in addition to the dichotomous test results (positive or negative). As none of the other readers provided quantifiable results, we were unable to compare signal intensity across the four UATs. The importance of the COI value in clinical routine has not been studied, and there are no previous studies using the STANDARD F for Legionella antigen detection.

Other aspects that were observed in the present study include the occurrence of invalid test results or problems with running the UAT that could not be resolved on two of the samples with FIA-based UATs. The sample that received an insufficient volume error with the STANDARD F UAT received the same error despite testing on new UAT cassettes as recommended by the manufacturer instructions. The manufacturer guidelines were given for errors in general, and did not have any further instructions on what could be done for 
this particular type of error. The sample did not appear to be viscous which would have affected the sample diffusion on the UAT. Additionally, the sample could be tested on the other UATs, and no issues were encountered when the same sample was tested separately on the STANDARD F S. pneumoniae UAT; hence, the reason for this occurrence is unknown. Additionally, this is the first time where all UATs were evaluated with their respective readers, and we observed that automated and visual interpretation of ICT-based UATs were mostly consistent with each other. For the ImmuView, we also noted that the samples with the strong positive Legionella signal interfered with the automated interpretation of pneumococcal antigen detection, resulting in a false positive result for pneumococcal antigen. This may be something that needs to be solved to avoid false positive results.

Information on how various UATs are utilized under clinical testing conditions are an important aspect to enable clinics to decide which UAT is most appropriate to implement in their diagnostic workflow with consideration of their specific needs and available resources. However, this is often not reported in UAT comparison studies. In the present study, comparing four UATs simultaneously, we had the unique opportunity to compare their user friendliness, and in this respect we observed that the FIA-based UATs have fewer steps required to setup samples, and hence less hands-on time. Despite requiring more sample setup procedures, the ICT-based UATs do have the flexibility to not require a reader for interpreting results. For the BinaxNOW, STANDARD F, and Sofia UATs, the availability of barcode scanners, connection to the laboratory information system (LIS), as well as the flexibility to choose between automated incubation followed by automated reading of samples, or reading of multiple samples in quick succession after a manually timed incubation also provides increased convenience for staff.

A limitation to our study is that we used frozen urine samples for evaluating UAT performance, and that sample numbers were small. We experienced difficulty obtaining more positive samples due to the current SARS-COV-2 pandemic. Another limitation is that the information we were able to retrieve from the LIS was limited to recent blood and respiratory culture results in relation to the urine sample, hence making it difficult to interpret the false positive results obtained.

In conclusion, we compared four Legionella UATs with automatic interpretation of test results using defined clinical samples and found that they performed similarly in detection of Legionella urinary antigen. Results between automated and visual interpretation of ICT-based UATs were mostly consistent with each other. The use of an automated reader also brings increased flexibility and convenience in performing urinary antigen assays in the clinical routine.

Supplementary Materials: The following are available online at https://www.mdpi.com/2076-2 607/9/3/493/s1, Table S1: Legionella UAT results for Legionella positive cases as interpreted by automatic readers, Table S2: Legionella UAT results for negative controls as interpreted by automatic readers.

Author Contributions: Conceptualization, and methodology, A.Y.W.W., A.I., S.A. and V.Ö.; formal analysis, A.Y.W.W.; investigation, A.Y.W.W. and A.T.A.J; resources, A.I., S.A. and V.Ö.; data curation, A.Y.W.W. and A.T.A.J.; writing—original draft preparation, A.Y.W.W.; writing—review and editing, A.Y.W.W., A.I., S.A., V.Ö.; supervision, project administration, and funding acquisition, S.A. and V.Ö. All authors have read and agreed to the published version of the manuscript.

Funding: This research and the APC was funded by the Vinnova Medtech4Health grant, grant number 2018-03896.

Institutional Review Board Statement: Ethical review and approval were waived for this study, due to the use of leftover samples that were anonymized. The test results were not sent to the clinicians and the patient charts were not analyzed.

Informed Consent Statement: Written informed consent has been obtained from the patient(s) were not necessary as described above.

Data Availability Statement: The data presented in this study are openly available upon request. 
Acknowledgments: We thank the blood culture section at the Department of Clinical Microbiology at Karolinska University Hospital Huddinge, Stockholm, Sweden, and Department of Laboratory Medicine, Örebro University Hospital, Örebro, Sweden for their help with sample collection. We also thank Özcan Ateş for his help with experiments. Readers for the four kits were provided by their respective manufacturers. The STANDARD F200 Analyzer was provided by SD Biosensor (Gyeonggi, Republic of Korea), DIGIVAL instrument by Abbott (Illinois, USA), Sofia Fluorescent Immunoassay Analyzer by Quidel Corporation (California, USA), and ImmuView Reader by SSI Diagnostica (Hillerød, Denmark). Assay kits for the study was purchased at discounted prices from their respective manufacturers.

Conflicts of Interest: The authors declare no conflict of interest, and the funders had no role in the design of the study; in the collection, analyses, or interpretation of data; in the writing of the manuscript, or in the decision to publish the results.

\section{References}

1. Centers for Disease Control and Prevention. National Notifiable Diseases Surveillance System, 2018 Annual Tables of Infectious Disease Data; CDC Division of Health Informatics and Surveillance: Atlanta, GA, USA, 2019.

2. European Centre for Disease Prevention and Control. Legionnaires' Disease. ECDC; Annual epidemiological report for 2018; European Centre for Disease Prevention and Control: Stockholm, Sweden, 2020.

3. Dooling, K.L.; Toews, K.-A.; Hicks, L.A.; Garrison, L.E.; Bachaus, B.; Zansky, S.; Carpenter, L.R.; Schaffner, B.; Parker, E.; Petit, S.; et al. Active Bacterial Core Surveillance for Legionellosis-United States, 2011-2013. Morb. Mortal. Wkly. Rep. (MMWR) 2015, 64, 1190-1193. [CrossRef] [PubMed]

4. Burillo, A.; Pedro-Botet, M.L.; Bouza, E. Microbiology and Epidemiology of Legionnaire's Disease. Infect. Dis. Clin. North Am. 2017, 31, 7-27. [CrossRef] [PubMed]

5. Pierre, D.M.; Baron, J.; Yu, V.L.; Stout, J.E. Diagnostic testing for Legionnaires' disease. Ann. Clin. Microbiol. Antimicrob. 2017, 16, 59. [CrossRef] [PubMed]

6. Athlin, S.; Iversen, A.; Özenci, V. Comparison of the ImmuView and the BinaxNOW antigen tests in detection of Strep-tococcus pneumoniae and Legionella pneumophila in urine. Eur. J. Clin. Microbiol. Infect. Dis. 2017, 36, 1933-1938. [CrossRef] [PubMed]

7. Jørgensen, C.S.; Uldum, S.A.; Sørensen, J.F.; Skovsted, I.C.; Otte, S.; Elverdal, P.L. Evaluation of a new lateral flow test for detection of Streptococcus pneumoniae and Legionella pneumophila urinary antigen. J. Microbiol. Methods 2015, 116, 33-36. [CrossRef] [PubMed]

8. Edelstein, P.H.; Jørgensen, C.S.; Wolf, L.A. Performance of the ImmuView and BinaxNOW assays for the detection of urine and cerebrospinal fluid Streptococcus pneumoniae and Legionella pneumophila serogroup 1 antigen in patients with Le-gionnaires' disease or pneumococcal pneumonia and meningitis. PLOS ONE 2020, 15, e0238479. [CrossRef] [PubMed]

9. Badoux, P.; Kracht-Kosten, L.; Herpers, B.; Euser, S. Method Comparison of the ImmuView L. pneumophila and L. longbeachae Urinary Antigen Test with the BinaxNOW Legionella Urinary Antigen Card for Detection of Legionella pneumophila Serogroup 1 Antigen in Urine. J. Clin. Microbiol. 2019, 58, 01429-19. [CrossRef] [PubMed]

10. Ito, A.; Yamamoto, Y.; Ishii, Y.; Okazaki, A.; Ishiura, Y.; Kawagishi, Y.; Takiguchi, Y.; Kishi, K.; Taguchi, Y.; Shinzato, T.; et al. Evaluation of a novel urinary antigen test kit for diagnosing Legionella pneumonia. Int. J. Infect. Dis. 2021, 103, 42-47. [CrossRef] [PubMed]

11. Helbig, J.H.; Uldum, S.A.; Lück, P.C.; Harrison, T.G. Detection of Legionella pneumophila antigen in urine samples by the BinaxNOW immunochromato-graphic assay and comparison with both Binax Legionella Urinary Enzyme Immunoassay (EIA) and Biotest Legionella Urin Antigen EIA. J. Med. Microbiol. 2001, 50, 509-516. [CrossRef] [PubMed]

12. Domínguez, J.; Galí, N.; Matas, L.; Pedroso, P.; Hernández, A.; Padilla, E.; Ausina, V. Evaluation of a Rapid Immunochromatographic Assay for the Detection of Legionella Antigen in Urine Samples. Eur. J. Clin. Microbiol. Infect. Dis. 1999, 18, 896-898. [CrossRef] [PubMed]

13. Diederen, B.M.; Peeters, M.F. Evaluation of Rapid U Legionella Plus Test, a new immunochromatographic assay for de-tection of Legionella pneumophila serogroup 1 antigen in urine. Eur. J. Clin. Microbiol. Infect. Dis. 2006, 25, 733-735. [CrossRef] [PubMed]

14. Chidiac, C.; Bru, J.-P.; Choutet, P.; Decazes, J.-M.; Dubreuil, L.; Leport, C.; Lina, B.; Perronne, C.; Pouchain, D.; Quinet, B. Management of lower respiratory tract infections in immunocompetent adults. Médecine Mal. Infect. 2006, 36, S262-S272.

15. Deforges, L.; Legrand, P.; Tankovic, J.; Brun-Buisson, C.; Lang, P.; Soussy, C.-J. Case of False-Positive Results of the Urinary Antigen Test forLegionella pneumophila. Clin. Infect. Dis. 1999, 29, 953-954. [CrossRef] [PubMed]

16. Pontoizeau, C.; Dangers, L.; Jarlier, V.; Luyt, C.E.; Guiller, E.; Fievet, M.H.; Lecso-Bornet, M.; Aubry, A.; Brossier, F. Ruling out False-Positive Urinary Legionella pneumophila Serogroup 1 and Streptococcus pneumoniae Antigen Test Results by Heating Urine. J. Clin. Microbiol. 2014, 52, 4347-4349. [CrossRef] [PubMed]

17. Beraud, L.; Gervasoni, K.; Freydiere, A.M.; Descours, G.; Ranc, A.G.; Vandenesch, F.; Lina, G.; Gaia, V.; Jarraud, S. Comparison of Sofia Legionella FIA and BinaxNOW ${ }^{\circledR}$ Legionella urinary antigen card in two national ref-erence centers. Eur. J. Clin. Microbiol. Infect. Dis. 2015, 34, 1803-1807. [CrossRef] 